\title{
To Study the Effect of BER and Q-factor in Intersatellite Optical Wireless Communication System
}

\author{
${ }^{1}$ A. K. Jaiswal, ${ }^{2}$ Anil Kumar, ${ }^{3}$ Santosh Tripathi, ${ }^{4}$ Amarendra Kumar Chaudhary \\ Shiats, Allahabad, India
}

\begin{abstract}
The system performance plays a great role in analyzing the characteristics of optical wireless communication link. This paper describes the effect of BIT ERROR RATIO and QUALITY FACTOR in communication system. The effect of diameter antennae with received power and distance is also analyzed in this paper. The value of antennae diameter varies from $20 \mathrm{~cm}$ to $40 \mathrm{~cm}$ and distance between satellites varies from $0 \mathrm{~km}$ to $5000 \mathrm{~km}$. The optical received power can be associated with the receivers sensitivity where increasing the received power would results in increasing the sensitivity.
\end{abstract}

Keywords: Intersatellite optical wireless communication (IsOWC), Q-factor, BER.

\section{Introduction}

In the communication world, satellite optical wireless communication (SOWC) is one of the major areas that remains to be comprehensively researched (1).Optical inter satellite links (ISL) provide an attractive alternate to microwave systems for both commercial and military applications. The advantages for optical ISL include higher band width, lower power requirements and smaller antenna size. An optical ISL operating at 850 $\mathrm{nm}$ wavelength using a $10 \mathrm{~cm}$ diameter transmitter telescope will have an effect angular bandwidth of $10 \mu$ radian compared to a typical microwave beam width of several milli radians(2). It is important in satellite optical communication to dissipate minimum power and to obtain minimum BER. This aim can achieved with very small transmitter divergence angles to assure maximum received power. The disadvantages of two narrow divergence angles in a simplistic manner are that the transmitter beam may sometimes miss the receiver satellite due to pointing vibrations. Also for small divergence angles, the transmitter optics aperture is big and expensive. The optimum value of the received power as a function of the pointing vibration displacement determines the optimum beam divergence angle. Such design reduces the price of the mission and increases the reliability of the system (3). The overall system performance of a communication link is easily quantified using a link budget, the techniques being similar to those used to evaluate microwave links. There are three important parameters: transmitter power, propagation losses, and receiver sensitivity. The receiver sensitivity relates the amount of optical power needed to maintain the signal to noise ratio required to achieve a desired quantity of service (5). This paper shows the effect of Q-factor on BER and also analyzes the dependency of received power on antenna diameter (4).

\section{System Performance}

The system performance can be evaluated in many ways such as by analyzing the BER and Q-factor. BER can be said to be the ratio of the number of bit errors detected in the receiver and the no. of bits transmitted. Bit error happens the results of incorrect decisions being made in a receiver due to the presence of noise on a digital signal (6). Meanwhile, Q-factor is a measurement of the signal quality. It is proportional to the systems signal to noise ratio. In optical system, the BER is typically too small to measure hence Q-factor is more suitable to be used. The relationship between BER and Q-factor can be given as

$\operatorname{BER}=1 / 2 \operatorname{erfc}(\mathrm{Q} / \sqrt{2}) \approx 1 / \sqrt{2} \pi \mathrm{Q} \exp \left(-\mathrm{Q}^{2} / 2\right)[7]$

The attenuation, $\mathrm{A}$, for intersatellite link and satellite to earth link is calculated using the equation $\mathrm{A}=\mathrm{L}^{2}\left(\lambda / \mathrm{D}_{\mathrm{T}}\right)^{2} / \mathrm{D}_{\mathrm{R}}{ }^{2} \mathrm{~T}_{\mathrm{R}} \mathrm{T}_{\mathrm{T}}\left(1-\mathrm{L}_{\mathrm{P}}\right)$

Where $L$ is the distance between the transmitter and receiver, $\lambda$ is the wavelength, $D_{R}$ is the receiver optical antenna diameter, $\mathrm{L}_{\mathrm{p}}$ is the pointing loss, $\mathrm{T}_{\mathrm{R}}$ and $\mathrm{T}_{\mathrm{T}}$ are the transmission factor for the transmitter and the receiver respectively , $\mathrm{D}_{\mathrm{T}}$ is the diameter of the transmitter optical antenna

According to Pfennigbauer[8] we take following values as follow

$\mathrm{D}=\mathrm{D}_{\mathrm{T}}=\mathrm{D}_{\mathrm{R}}, \mathrm{L}_{\mathrm{P}}=.2, \mathrm{~T}_{\mathrm{R}}=\mathrm{T}_{\mathrm{T}}=0.8, \lambda=1550 \mathrm{~nm}$

Thus the propagation atteneuation, $\mathrm{A}$, for inter satellite optical link is given as $\mathrm{A}=4.69 * 10^{-22} \mathrm{~L}^{2} / \mathrm{D}^{4}$ 


\section{Result And Discussion}

$\mathrm{Q} \uparrow \mathrm{BER} \downarrow$

We know Q- factor is a measurement of the signal quality. For the performance of any system higher value of Q-factor is required. Since the BER is inversely proportional to Q-factor. Therefore if the system error increases the Q-factor will thus decrease. So to reduce the BER we must increase the Q-factor. In optical system the BER is typically too small to measure, hence Q-factor is more suitable to be used.

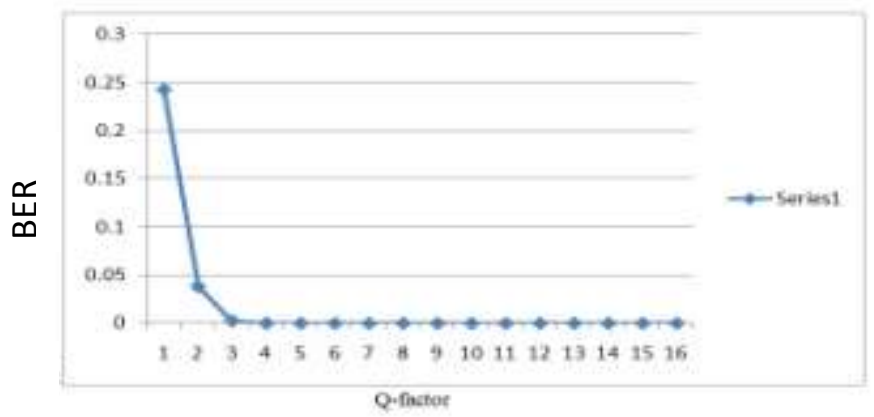

(2) $\quad \mathrm{L} \uparrow \mathrm{A} \uparrow \operatorname{Pr} \downarrow$

When the distance between the transmitter and receiver increases, keeping the diameter of optical antennae constant, the Attenuation go on increasing. We know that $\mathrm{Pr}=\mathrm{Pi}-\mathrm{A}$, hence if attenuation will be more then received power will be less.

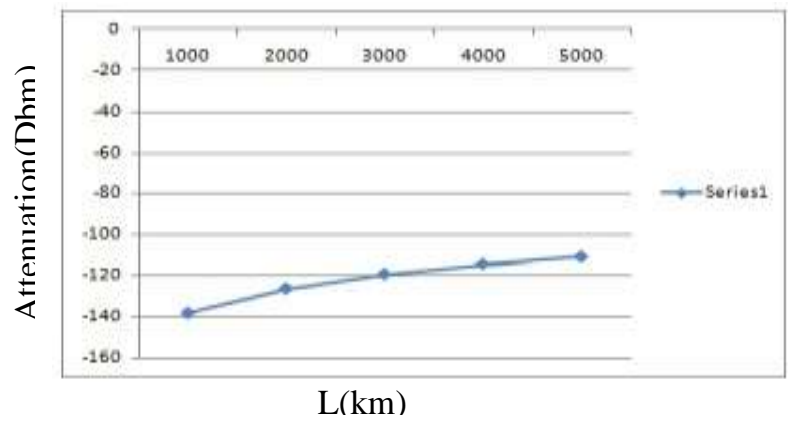

(3) $\quad \mathrm{D} \uparrow \mathrm{A} \downarrow \operatorname{Pr} \uparrow$

By keeping distance between transmitter and receiver constant if we increase diameter of optical antennae, then Attenuation will reduce, and hence received power will be increases.

$\mathrm{D}(\mathrm{cm})$

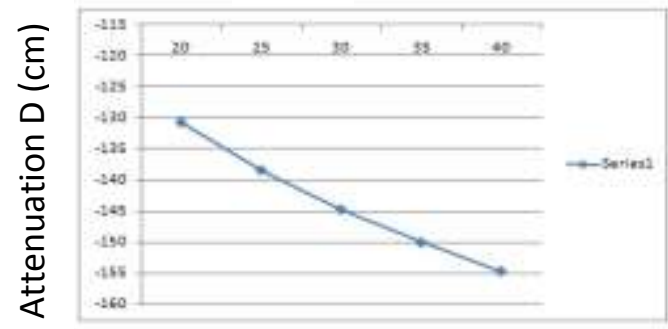

\section{Conclusion}

In this discussion we have seen that the BER is inversely proportional to Q-factor. So it can be concluded that signals with smaller bit rate travel more than the one with higher bit rate. The received power for the system is the difference between the input power and the attenuation in decibels. The power received is observed to be increasing with the increment of optical antennae diameter. However, increasing the distance between the satellite reduce the level of received power. Optical received power can be associated with the receivers sensitivity where increasing the received power would result in increasing the sensitivity. Therefore it can be concluded that by increasing the diameter optical antennae, the receiver sensitivity is also increased. It 
To Study the Effect of BER and Q-factor in Intersatellite Optical Wireless Communication System can also be confirmed that lower sensitivity is obtained for longer inter-satellite distance, Higher sensitivity will allow more throughputs and reducing the error received by the system.

\section{Refrerences}

[1] ShlomiArnon, Optimization of Urban Optical Wireless Communication systems, ieee transactions on wireless communications, vol. 2, no. 4, july 2003

[2] Chien-Chung Chen, Chester S. Gardner, Impact of Random Pointing and Tracking Errors on the Design of Coherent and Incoherent Optical Intersatellite Communication Links, IEEE Transaction On Communication, Vol. 37, No. 3, March 1989

[3] S. arnon ,S. rotman, n. S. kopeika, Optimum Transmitter Optics Aperture for Satellite Optical Communication ieee transactions on aerospace and electronic systems vol. 34, no. 2 april 1998.

[4] Kuldeep Singh, Dr.Manjit Singh Bhamrah, Investigation on Aperture Diameter of Telescope in Intersatellite Optical Wireless system., Vol. 2, Issue 3, May-Jun 2012, pp. 1666-1670.

[5] Anthony Acampora, Scott H. bloom, Srikanth Krishnamurthy, UniNet:A Hybrid Approach for Universal Broadband Access Using Small Radio Cells Interconnected by Free-Space Optical Links, IEEE JOURNAL On Selected Areas In Communication, Vol..16, No. 6, August 1998

[6] Aida HasfizaHashim, Farah Diana Mahad, Sevia M. Idrus and Abu Sahmah, M. Supa'at, Modeling and Performance Study of InterSatellite Optical Wireless Communication System, University of Photonics Technology Centre, Faculty of Electrical Engineering Malaysia ,2010.

[7] Marcuse, D., Calculation of Bit-Error Probability for a Lightwave System with Optical Amplifier and Post-Detection Guassion Noise. Journal of Lightwave Technology, 1991 .9(4):505-513

[8] Pfennigbauer, M. and Leeb, W.R. Free-Space Optical Quantum key distribution using Intersatellite Links. CNES - Intersatellite Link Workshop. November 2003 